\title{
Laminar forced convection heat transfer in microcapsulated phase change material suspensions
}

\author{
MANISH GOEL, $\dagger$ S. K. ROY $\dagger$ and S. SENGUPTA $\ddagger$ \\ $\dagger$ Department of Mechanical Engineering, University of Miami, Coral Gables, FL 33124, U.S.A. \\ $\ddagger$ School of Engineering, University of Michigan, Dearborn, MI 48128, U.S.A.
}

(Received 22 July 1992 and in final form 27 August 1993)

\begin{abstract}
An experimental study using a suspension of $n$-eicosane microcapsules in water was conducted in order to evaluate the heat transfer characteristics of phase change material suspensions. Experiments were done for laminar, hydrodynamically fully developed flows in a circular duct with a constant wall heat flux. The temperature of the suspension entering the test section was maintained at or below the melting point of the phase change material. The primary parameters in the study were the bulk Stefan number and the volumetric concentration. In addition, a few experiments were conducted to evaluate the effect of particle diameter and degree of homogeneity of the suspension. The heat fluxes chosen for the experiments were typical of low temperature applications (below $60^{\circ} \mathrm{C}$ ). Results show that use of phase change material suspensions can reduce the rise in wall temperature by up to $50 \%$ as compared to a single phase fluid for the same non-dimensional parameters. The most dominant parameter influencing the heat transfer was found to be the bulk Stefan number. The effect of concentration was found to be insignificant by itself, though its influence is felt indirectly through the bulk Stefan number. An increase in particle diameter by a factor of 2.5 was found to further reduce the wall temperature rise by $15 \%$. The degree of homogeneity of the suspension had no observable effect on the wall temperatures.
\end{abstract}

\section{INTRODUCTION}

THE USE of microencapsulated phase change suspensions as heat transfer fluids has been suggested in recent years. These suspensions benefit from a number of special features including (a) a high energy storage density due to the absorption of latent heat during the phase change process, (b) relatively low variations in operating temperatures of systems using such fluids due to energy absorption at approximately constant temperature, (c) the possibility of using the same medium for both energy transport and storage, thereby reducing losses during the heat cxchange process, (d) lower pumping power requirements due to the increased heat capacity [1], (e) high heat transfer rates to the phase change material due to large surface area to volume ratio, (f) the enhanced thermal conductivity of suspensions leading to increased heat transfer to the suspension [2], and (g) the reduction/elimination of incongruent melting and phase separation [3].

Mehalick and Tweedie [4] introduced the concept of a carrier fluid for encapsulated particles of phase change materials, and Bahtami [5] later studied the feasibility of their use at high temperature and heat fluxes. Hart and Thornton [3] reported a two-fold increase in the effective specific heat of a suspension of $30 \%$ wax capsules in oil tested in a prototype solar collector pump at a temperature range of $100-120^{\circ} \mathrm{C}$. They also showed that microcapsules ranging in diameters from 5-50 $\mu \mathrm{m}$ can be pumped without significant damage. McMahon et al. [6] carried out thermal performance tests of slurries of $n$-heptadecane and $n$ octadecane in water-ethylene glycol as cooling fluids for protective garments and reported an effective specific heat increase of $20 \%$. They reported better results with $n$-octadecane but also recognized that phase separation could reduce the heat transfer capability of the suspension. Kasza and Chen [1] estimated that the heat transfer coefficient could be increased by up to three times by the use of microencapsulated phase change suspensions. Colvin and Mulligan $[7,8]$ and Colvin et al. [9] reported specific heat increases up to 5 times and heat transfer coefficient increases of up to 2.8 times in flows of microencapsulated phase change material suspensions, and suggested applications in avionics, spacecraft and electronic systems. Chen and Chen [10] developed a model for heat transfer augmentation using phase change suspension flows over flat plates and conducted experiments to test the validity of the model. They reported excellent agreement in their experimental and theoretical results which showed as much as threefold incrcase in the heat transfer coefficient. Colvin and Mulligan [11] have also obtained a patent for closed loop cooling systems with the heat source and sink physically close to each other and with both temperatures near the melting point of the phase change material. Under these circumstances, significant heat transfer enhancements and reductions in pumping powers can be obtained.

Charunyakorn et al. [12,13] conducted a numerical simulation of microencapsulated phase change suspension flow in circular tubes and parallel plates for 


\section{NOMENCLATURE}

c. volumetric concentration

$C$ specific he'd

d diameter

$k$ thermal conductivity

$l$ length

$L \quad$ latent heat of phase change material

$\operatorname{Pr} \quad$ Prandtl number

$q \quad$ heat flux

$Q$ heat input

$r$ radius

Re Reynolds number

Ste Stefan number

$t$ time

$T$ temperatures

$U$ flow velocity

$V$ volume

$x \quad$ axial distance

$\bar{x} \quad$ non-dimensional axial distance (equalions (7), (8)).
Greek symbols

$x$ thermal diffusivity

0) non-dimensional temperature (equations (7), (8))

$\mu \quad$ dynamic viscosity

$v \quad$ kinematic viscosity

$\rho$ density.

Subscripts

111 relating to thermocouple locations on the test section

b bulk fluid

c phase change material core

corr with sensible heat correction

d duct

f fluid

i inlet

$\mathrm{m} \quad$ mean, melting point

$\mathrm{p}$ particle

w wall

$x$ at axial position $x$ different boundary conditions for low temperature applications. Their parametric study shows that the phase change material heat transfer in suspension flows is dependent on the bulk Stefan number and volumetric particle concentration. Their model also predicts augmentation of heat transfer coefficient by 1.5-3 times combined with a $40-60 \%$ reduction in temperature rise.

Studies on natural convection systems were conducted by Katz [14] and Datta et al. [15]. Katz [14] used suspensions of phase change material encapsulated in relatively large capsules with diameters of the order of a few millimeters and showed that heat transfer could be enhanced by about 2 times. Datta et al. [15] have done further work using microencapsulated phase change suspensions and reported up to $80 \%$ increase in heat transfer for up to $5 \%$ concentrations. Heat transfer reduced at higher concentrations due to agglomeration and settling effects.

\section{Scope of study}

Earlier studies suggest that up to a three-fold enhancement in heat transfer coupled with at least a $50 \%$ reduction wall temperature rise can be obtained by using a microencapsulated phase change material suspension as the heat transfer fluid. Though a great deal of experimental data have been reported by Colvin and Mulligan [7] and Colvin et al. [9], much of these are presented in a form not directly usable by engineers and researchers not involved in those projects. In addition, no systematic experimental data suitable for design are available in the literature. The present work on laminar forced convection heat trans- fer with suspensions of microencapsulated phase change materials is a first step towards that end. Experiments have been conducted to evaluate laminar heat transfer in a circular duct with a constant wall heat flux boundary condition. The suspension enters the test section at or below the melting temperature of the phase change material in order to ensure that all phase change takes place in the test section.

\section{EXPERIMENTAL INVESTIGATION}

\section{Selection of suspension}

Properties desired in a phase change material for use in a phase change material suspension are similar to those necessary for energy storage, i.e. it should have (a) a high latent heat of fusion, (b) a high specific heat and thermal conductivity, and (c) a high density and a low volume change during phase change. Of these, a high latent heat of fusion is of primary importance since the heat transfer enhancement is mostly due to energy storage in the heat transfer medium [12. 13]. In contrast, the contributions of the other factors are relatively small.

For practical applications, ease and economy of microencapsulation, thermal stability and structural integrity of the microcapsules are also very important in addition to the above properties $[3,6,8]$. In a previous study [16], microcapsules of $n$-eicosane $\left(\mathrm{C}_{20} \mathrm{H}_{42}\right)$ in an amino formaldehyde resinous wall were evaluated for use in microencapsulated phase change suspensions. Since these microcapsules were found to be structurally and thermally stable, they were used as the suspended phase in the present study. The properties of the phase change material and the 
wall of the microcapsules are given in Table 1. Further details regarding the microcapsules are given in ref. [16].

Two main criteria used in the selection of a fluid as an energy transport medium are that it should have a high thermal conductivity and specific heat. However, once $n$-eicosane was selected as the dispersed medium in the phase change suspension, the selection of the suspending fluid was governed by a more important factor-its compatibility with $n$-eicosane and the microcapsule wall. Water was chosen as the suspending fluid because it is easy to handle and has no effect on the phase change material or the microcapsule wall. This factor is very important since the suspension had to be separated and re-mixed a number of times during the experiments. In addition, the density of the microcapsules, though not identical to that of water, is quite close, so that a relatively homogeneous suspension could be obtained. Some experiments were also conducted at the end of the study by using $80 \%$ by volume aqueous ethanol solution to obtain an entirely neutrally buoyant suspension in order to evaluate the effects of nonhomogeneity.

\section{Microcapsule properties}

The phase change material, n-eicosane, made up about $70 \%$ of a microcapsule by weight [16]. Since the thermophysical properties of the $n$-eicosane and the wall material are different, the properties of the microcapsules must be calculated by considering the properties of the individual components. The density and specific heat of the microcapsules were calculated using mass and energy balance respectively, where the density of $n$-eicosane was taken as the mean of its solid and liquid densities [17]:

$$
\begin{gathered}
\rho_{\mathrm{p}}=\frac{10}{7}\left(\frac{d_{\mathrm{c}}}{d_{\mathrm{p}}}\right)^{3} \rho_{\mathrm{c}} \\
C_{\mathrm{p}}=\frac{\left(7 C_{\mathrm{c}}+3 C_{\mathrm{w}}\right) \rho_{\mathrm{c}} \rho_{\mathrm{w}}}{\left(3 \rho_{\mathrm{c}}+7 \rho_{\mathrm{w}}\right) \rho_{\mathrm{p}}} .
\end{gathered}
$$

The thermal conductivity of the microcapsule was calculated using the composite sphere approach. The thickness of the microcapsule wall determined the heat transfer resistance of the wall material, while the heat transfer resistance of the core material was evaluated considering the model for a solid sphere in an infinite medium [18]. Thus, the thermal conductivity of the microcapsule is given by:

$$
\frac{1}{k_{\mathrm{p}} d_{\mathrm{p}}}=\frac{1}{k_{\mathrm{c}} d_{\mathrm{c}}}+\frac{d_{\mathrm{p}}-d_{\mathrm{c}}}{k_{\mathrm{w}} d_{\mathrm{p}} d_{\mathrm{c}}} .
$$

Table 1 gives the property values of the microcapsules calculated using the above equations.

\section{Suspension properties}

The bulk suspension properties are a combination of the properties of the suspending fluid and the microcapsules. Using a mass and energy balance [17], the density and specific heat are:

$$
C_{\mathrm{h}}=\frac{c \rho_{\mathrm{p}} C_{\mathrm{p}}+(1-c) \rho_{\mathrm{f}} C_{\mathrm{f}}}{\rho_{\mathrm{b}}}
$$

To calculate the viscosity of the suspension, the following relation by Vand [19] was used :

$$
\frac{\mu_{\mathrm{b}}}{\mu_{\mathrm{f}}}=\left(1-c-1.16 c^{2}\right)^{-2.5}
$$

This relation has been shown to be valid for concentrations up to $20 \%$ and duct-to-particle diameter ratios of 20-100 and mean particle diameters of $0.3-$ $400 \mu \mathrm{m}$ (e.g. ref. [20]).

Maxwell's [21] relation for the bulk thermal conductivity of static dilute suspensions with conductivities of the same order for the suspending and the dispersed media was used to calculate the suspension conductivity. Yamada and Takahashi [22] found excellent agreement between this relation and their experimental values for suspensions of spherical particles at concentrations up to $22 \%$ :

Table 1. Physical properties of suspension components and suspensions

\begin{tabular}{lcccc}
\hline & $\begin{array}{c}\text { Specific } \\
\text { heat } \\
\left(\mathrm{J} \mathrm{kg}^{-1} \mathrm{~K}^{-1}\right)\end{array}$ & $\begin{array}{c}\text { Thermal } \\
\text { conductivity } \\
\left(\mathrm{W} \mathrm{m}^{-1} \mathrm{~K}^{-1}\right)\end{array}$ & $\begin{array}{c}\text { Kinematic } \\
\text { viscosity } \\
\left(\mathrm{m}^{2} \mathrm{~s}^{-1}\right)\end{array}$ \\
\hline $\begin{array}{l}\text { Water } \\
80 \% \text { aqueous ethanol } \\
\text { solution [25] }\end{array}$ & 997 & 4180 & 0.606 & $9.07 \times 10^{-7}$ \\
$\begin{array}{c}n \text {-Eicosane } \\
\text { (PCM core) [26] }\end{array}$ & 850 & 3098 & 0.25 & $1.25 \times 10^{-6}$ \\
$\begin{array}{c}\text { Urea-formaldehyde } \\
\text { (PCM wall) [27] }\end{array}$ & 778 (solid) & 2210 & 0.15 & $5.25 \times 10^{-8}$ \\
$\begin{array}{c}\text { PCM microcapsule } \\
5 \% \text { suspension }\end{array}$ & 1500 & 1672 & 0.42 & - \\
$\begin{array}{l}10 \% \text { suspension } \\
\text { 15\% suspension }\end{array}$ & $946.4 \dagger$ & 1973.1 & 0.150 & $5.25 \times 10^{-8}$ \\
$20 \%$ suspension & 983 & 4098 & 0.576 & $1.04 \times 10^{-6}$ \\
\hline
\end{tabular}

†Density of PCM used here is the mean of its solid and liquid densities. 


$$
\frac{k_{\mathrm{b}}}{k_{\mathrm{f}}}=\frac{2+\frac{k_{\mathrm{p}}}{k_{\mathrm{f}}}+2 c\left(\frac{k_{\mathrm{p}}}{k_{\mathrm{f}}}-1\right)}{2+\frac{k_{\mathrm{p}}}{k_{\mathrm{f}}}-c\left(\frac{k_{\mathrm{p}}}{k_{\mathrm{f}}}-1\right)} .
$$

The properties of the suspension according to equations (7)-(10) are listed in Table 1.

\section{Parameters of interest}

The parameters affecting the heat transfer in microencapsulated phase change material suspensions were identified as follows $[12,13]$.

a. Microcapsule concentration. A higher concentration of microcapsules signifies a higher energy storage capability of the suspension. However, it also leads to increased viscosity of the suspension and at high concentrations, non-Newtonian behavior. Concentrations used in this study were $5,10,15$ and $20 \%$ by volume of the suspension.

b. Bulk Stefan number. The bulk Stefan number is defined as the ratio of the sensible heat capacity of the suspension to its latent heat capacity $\left(\right.$ Ste $_{\mathrm{b}}=$ $\left.\left(C_{\mathrm{pb}}\left(q_{\mathrm{w}} r_{\mathrm{d}} / k_{\mathrm{b}}\right)\right) /\left(c L\left(\rho_{\mathrm{p}} / \rho_{\mathrm{b}}\right)\right)\right)$. For constant wall heat flux, the sensible heat capacity of the suspension is defined in terms of the absolute value of the characteristic temperature. Bulk Stefan numbers of 0.5, 1.0, 2.0 and 3.0 were considered in this study.

c. Duct-lo-particle diameter ratio. The avalability of $n$-eicosane microcapsules in two sizes, 100 and 250 $\mu \mathrm{m}$ also allowed a limited study of this parameter. Duct-to-particle diameter ratios of 12.6 and 31.4 have been considered for concentrations of 5 and $10 \%$ concentrations. Due to limited volumes of microcapsules available, experiments for higher concentrations were done with a mixture of microcapsules of both sizes.

d. Degree of homogeneity of the suspension. In their model, Charunyakorn et al. [13] considered the suspension to be homogeneous as it flowed through the test section. In this investigation, the suspension was not neutrally buoyant since the microcapsules were slightly ligher than water. Though a homogeneous suspension was obtained by thorough mixing before the suspension was forced into the test section, some separation of the microcapsules was seen to occur by the time it exited the test section. However, the degree of separation of the microcapsules is smaller for higher flow rates due to the lower residence time in the test section. Thus, the effect of the homogeneity of the suspension was studied by considering two flow rates corresponding to Reynolds numbers of approximately 200 and 1000 . Once again, due to the limited amount of microcapsules available, experiments were conducted for a volumetric concentration of $5 \%$ only. In addition, experiments were conducted for a neutrally buoyant suspension of $n$-eicosane in $80 \%$ by volume aqueous ethanol solution to further evaluate the effects of homogeneity of the suspension.

Two other parameters identified by Charunyakom et al. [13] that were not considered were the particleto-fluid thermal conductivity ratio and modified particle Peclet number. However, their analysis suggested their effects were negligible. Table 2 lists the experiments that were conducted based on the above parameters.

\section{Experimental apparatus}

Figure 1 shows a schematic diagram of the experimental set up. The test section was a circular copper tube, $0.3 \mathrm{~m}$ in length with an internal diameter of $3.14 \mathrm{~mm}\left(0.016^{\prime \prime}\right)$ and an outer diameter of $4.76 \mathrm{~mm}$ $\left(0.125^{\prime \prime}\right)$. Eleven T-type insulated copper-constantan thermocouples were silver soldered to the test section for measurement of wall temperatures along the axial direction. The thermocouples were split diametrically to use the copper tube as their hot junction. Constant heat flux was maintained at the test section by winding a 33 A.W.G. (0.20 $\mathrm{mm}$ diameter $)$ insulated copper wire around the copper tube. The insulation can withstand temperatures up to $150^{\circ} \mathrm{C}$. The wire resistance was $1.11 \Omega \mathrm{m}$, and $14.6 \mathrm{~m}$ of wire was wound on the tube ( 1475 closely packed turns) to provide a total resistance of $16.2 \Omega$. A DC power supply, rated at 30 V. I A max., was used to control the voltage to the wire and, thus, the heat input to the test section. A Plexiglas tube provided rigidity to the whole test section assembly. Fiber glass wool of thickness $1.15 \mathrm{~cm}$ was placed in the annulus between the test section and

Table 2. Table of experiments

\begin{tabular}{|c|c|c|}
\hline Parameter & $\begin{array}{l}\text { Parametric } \\
\text { values }\end{array}$ & Comments \\
\hline Concentration & $0.05,0.1,0.15,0.2$ & $\begin{array}{l}\text { Volumetric concentration of microcapsules in solution: } \\
\text { approximately } 70 \% \text { of microcapsules by weight is } n \text {-eicosane }\end{array}$ \\
\hline Bulk Stefan number & $0.5,1.0,2.0,3.0$ & $\begin{array}{l}\text { Stefan no. is a function of concentration } S t e_{\mathrm{b}}=0.5 \text { for } \\
c=0.05 \text {, and } S t e_{\mathrm{b}}=3.0 \text { for } c=0.15,0.20 \text { not included in } \\
\text { test matrix }\end{array}$ \\
\hline Particle diameter & $100,250 \mu \mathrm{m}$ & $\begin{array}{l}\text { Experiments with } 250 \mu \mathrm{m} \text { were conducted for } c=0.05 \\
\text { and } 0.1 \text { only }\end{array}$ \\
\hline Reynolds number & $\sim 200, \sim 1000$ & $\begin{array}{l}\text { Experiments for } R e \simeq 1000 \text { were conducted for } 5 \% \\
\text { concentration only }\end{array}$ \\
\hline Suspending fluid & Water, $80 \%$ aq. ethanol solution & $\begin{array}{l}80 \% \text { aqueous ethanol solution provided a neutrally } \\
\text { buoyant suspension only } 2 \text { cxpcriments were } \\
\text { conducted for this suspension: Ste }=0.5,1.0 \text { for } c=0.15\end{array}$ \\
\hline
\end{tabular}




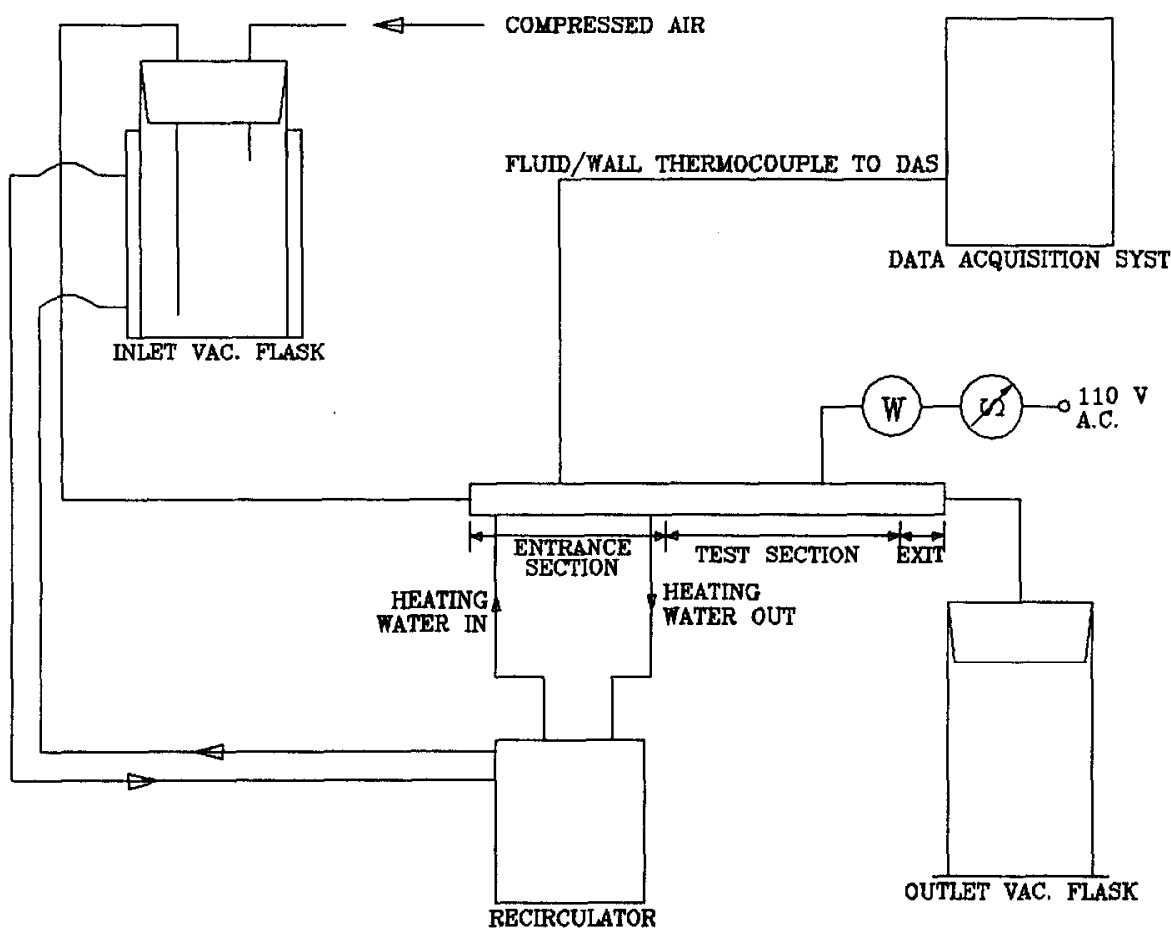

FIG. 1. Schematic diagram of the experimental setup.

a Plexiglas tube for this purpose. The Plexiglas tube was covered by aluminum foil on the outside and three silicone rubber guard heaters, $12.7 \mathrm{~cm} \times 12.7 \mathrm{~cm}$ $\left(5^{\prime \prime} \times 5^{\prime \prime}\right)$ in size. The temperatures on the inside of the guard heaters were measured at five locations along the length. A $13 \mathrm{~mm}\left(\frac{1}{2}\right)$ thick air conditioning foam insulation was provided around the guard heaters to further control the heat losses. To ensure that the flow entering the test section was developed, a Plexiglas entrance section, $500 \mathrm{~mm}$ long, was provided upstream of the test section. The temperature of the entering suspension was measured using an Omega HYP-0 T-type hypodermic thermocouple probe (33 A.W.G., $1^{\prime \prime}$ long needle) $15 \mathrm{~mm}$ ahead of the interface. Diametrically opposite to the hypodermic thermocouple, another thermocouple was placed to measure the wall temperature. The entrance section was also provided with a water jacket to ensure that the suspension entered the test section at or below the melting temperature of $n$-eicosane. The other end of the entrance section was connected to the suspension inlet reservoir, which was an Erlenmeyer flask placed in a constant temperature bath and provided with a thermocouple for measuring the suspension temperature, and the suspension was forced into the tube using compressed air. The inlet reservoir was placed on a magnetic stirrer to ensure homogeneity of the suspension as it entered the entrance section. The heating water to the water jacket and the bath for the inlet reservoir was recirculated by means of a Lauda RKS-20D recirculating bath. A Plexiglas exit section, $20 \mathrm{~mm}$ long, was provided downstream of the test section, which ended in a receiving flask. A thermocouple was placed $10 \mathrm{~mm}$ downstream of the interface to measure the wall temperature. All thermocouples were 30 A.W.G. insulated T-type copper-constantan thermocouples, except the T-type hypodermic thermocouple for measuring the inlet suspension temperature. The thermocouples were connected to a Fluke 2280B datalogger which recorded information on an IBM PC-AT by means of an RS-232 interface and the Fluke Prologger Communications software. The datalogger provided for ice point compensation required for measuring temperatures using thermocouples. For each set of data recorded, all thermocouples were scanned five times continuously during a one minute interval and the average temperatures from the five scans for any individual thermocouple were directly recorded.

\section{Calibration and verification}

Thermocouples were calibrated against known temperatures in the LAUDA RKS-20D recirculator which could maintain temperature accuracies of $\pm 0.03^{\circ} \mathrm{C}$. The circulator was programmed to vary the bath temperature from 20 to $70^{\circ} \mathrm{C}$ after every fifty minutes and temperatures were recorded every minute. The checkpoints used were $20,30,40,50,60$, and $70^{\circ} \mathrm{C}$ with a changeover time of ten minutes between two checkpoints. The average calibration curve was the straight line which fitted the scattered calibration data points using the least squares criterion [23]. The maximum error of calibration of any thermocouple was $\pm 0.03^{\circ} \mathrm{C}$. Flow rate checks were done to ensure con- 
stant flow rate was being maintained. It was observed that flow rates could be maintained constant to an accuracy of $\pm 10 \%$.

The apparatus was verified using water as the heat transfer fluid. Heat transfer runs were carried out to cover the whole range of heat inputs and temperatures that were used in the suspension experiments. Experimentally measured values of wall temperature as a function of the axial distance along the tube were compared in a non-dimensional form to analytical solutions for heat transfer in a single phase fluid flow through a circular tube constant wall heat flux. The wall temperature and axial distance along the tube were non-dimensionalized as follows:

$$
0_{\mathrm{wx}}=\frac{T_{\mathrm{wx}}-T_{\mathrm{i}}}{\frac{q_{\mathrm{w}} r_{\mathrm{d}}}{k_{\mathrm{r}}}}, \quad \overline{\mathrm{x}}=\frac{x}{r_{\mathrm{d}} \operatorname{Re} \operatorname{Pr}}
$$

where $x$ is the distance along the tube, $r_{\mathrm{d}}$ the duct radius, $q_{w}$ the wall heat flux, $T_{w}$ and $T_{1}$ the wall and inlet temperatures respectively, the Prandtl number $\operatorname{Pr}=\mu_{\mathrm{b}} C_{\mathrm{pb}} / k_{\mathrm{b}}$ and the Reynolds number $R e=$ $U_{\mathrm{m}} d_{\mathrm{d}} / v$. In the definition of the Reynolds number and the Prandtl number, $U_{\mathrm{m}}$ is the average velocity, $d_{\mathrm{d}}$ the duct diameter, $v$ the kinematic viscosity, $\mu$ the dynamic viscosity, $C_{\mathrm{p}}$ the specific heat, $k$ the thermal conductivity, and the subscript b refers to the bulk properties.

The results of the validation experiments are shown in Fig. 2. The maximum deviation with respect to the analytical solution can be seen to be of the order of $20 \%$. A careful evaluation of the experimental apparatus and the experiments showed no obvious reason (such as mixed convection effects, etc.) for this discrepancy. Though errors of this magnitude are quite common in heat transfer experiments, the apparent bias or systematic error near the central portion of the tube (thermocouples 6-8) has the unfortunate effect of lowering the temperatures in this region. In the absence of a systematic error correction, the effects of phase change (lower wall temperatures during the melting process) would be enhanced by this error, which would lead to excessively optimistic predictions. The use of such a correction will give a more conservative estimate for temperature control applications. Keeping this in mind, a systematic crror correction has been applied to all the results presented in this paper. Details of the correction, as well as results without this correction are given in Goel [17]. Figure 3 shows the verification results after the systematic errors were corrected as above.

\section{RESULTS AND DISCUSSIONS}

Figures 4-9 summarize the significant results obtained in this study. These are presented as the nondimensional wall temperature vs the non-dimensional axial distance along the tube as was the case for the pure fluid verification runs. A curve for heat transfer with a single phase fluid is also presented so that the standard single phase results may be compared with those of the phase change material suspension. No results are provided for the heat transfer coefficient or the Nusselt number. The reason for this has to do with problems in defining a suitable heat transfer coefficient when phase change suspensions are used. For pure fluids, the heat transfer coefficient is defined based on the local temperature difference $\left(T_{w}-T\right.$ (bulk, fluid)). If no change of phase is involved, the bulk fluid temperature at any location $x$ can be readily calculated by taking the overall heat balance. When phase change occurs, the local bulk mean temperature can be defined either as the 'mixed-mean' temperature (i.e. the temperature of the fluid at any cross-section if the fluid was completely mixed at that cross-section) or as the mathematical average of the temperatures at

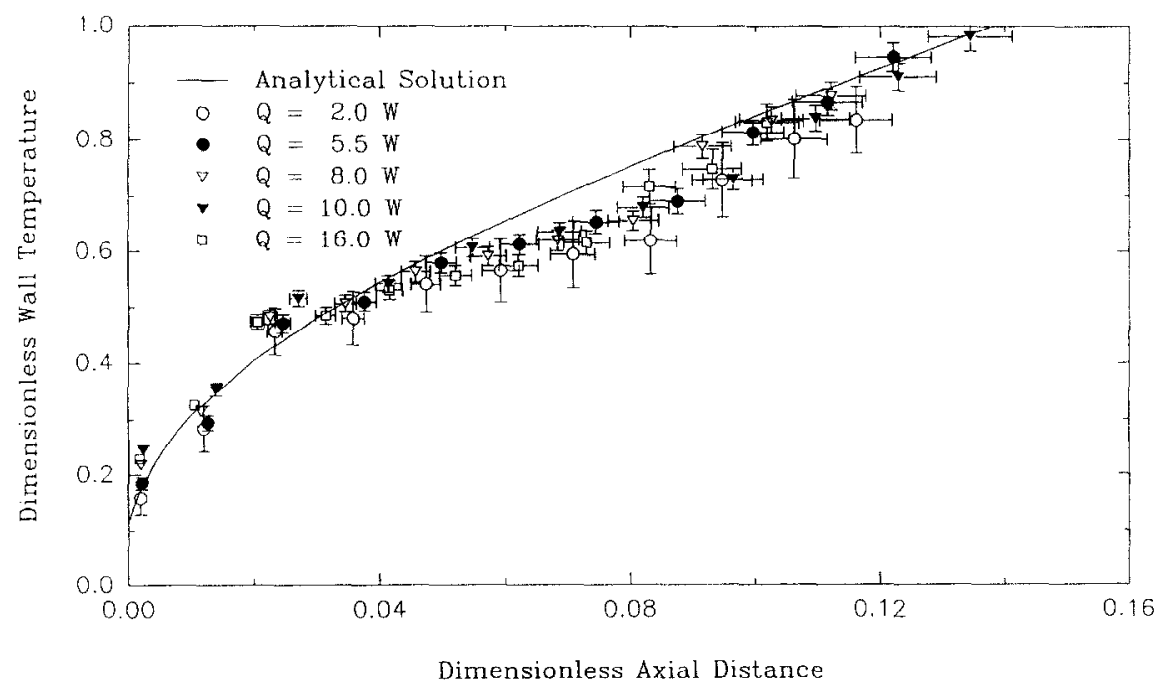

FIG. 2. Verification results for the experimental apparatus (heat transfer fluid: water). 


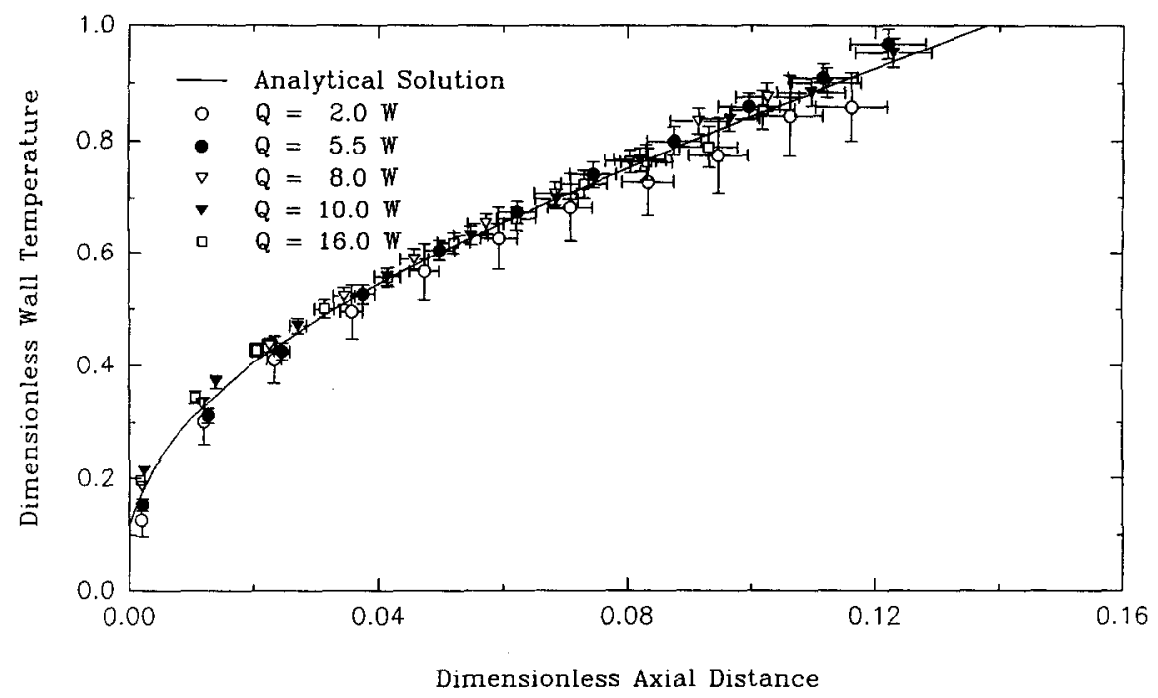

FIG. 3. Verification results for the experimental apparatus : data corrected for systematic errors.

any cross-section. Though these are equivalent for pure fluids, they can be quite different for phase change suspensions. For example, for cases with zero subcooling, the mixed-mean temperature can be equal to the phase change temperature even when local temperatures in the fluid are greater than the melting temperature, whereas the mathematical average temperature will always be greater than the melting temperature except at the inlet. Both definitions have their advantages and disadvantages, with the first having a stronger physical basis, and the second being more useful from a computational viewpoint (e.g. [13]), but there does not seem to be a consensus on this matter. In any case, if the second (mathematical average) approach is used, temperatures must be measured at different points in the flow field at every cross-section. Since temperature control was the primary application for this study, such measurements were not made. For temperature control applications, the Nusselt numbers based on the bulk (physical) mean temperatures are not of any significance, and are therefore not presented in this paper.

\section{Effect of Stefan number}

Figure 4 shows the effect of Stefan number on the wall temperatures for given concentrations. The results are also compared to Charunyakorn et al.'s [13] predictions. For a given concentration, the wall

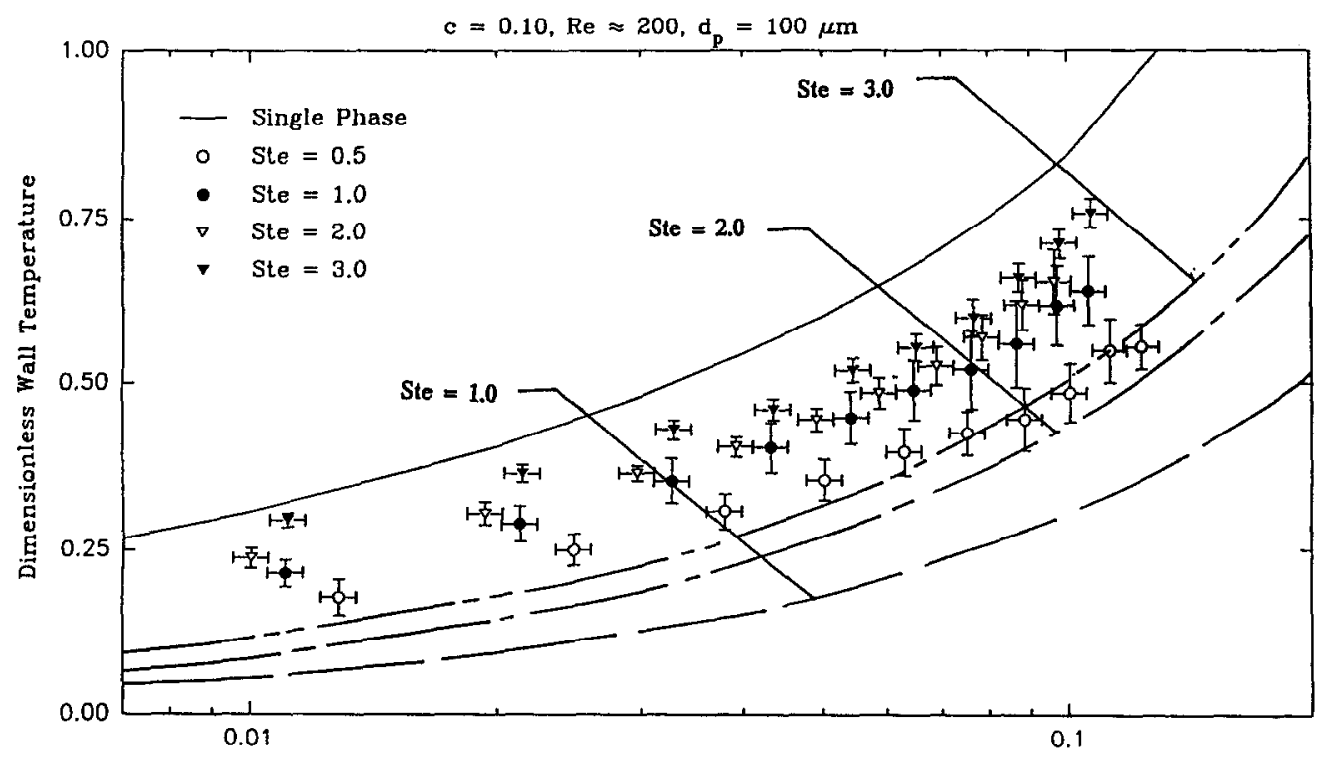

Fig. 4. Effect of bulk Stefan number on wall temperatures: comparison with numerical predictions from ref. [13] for $c=0.1$ 


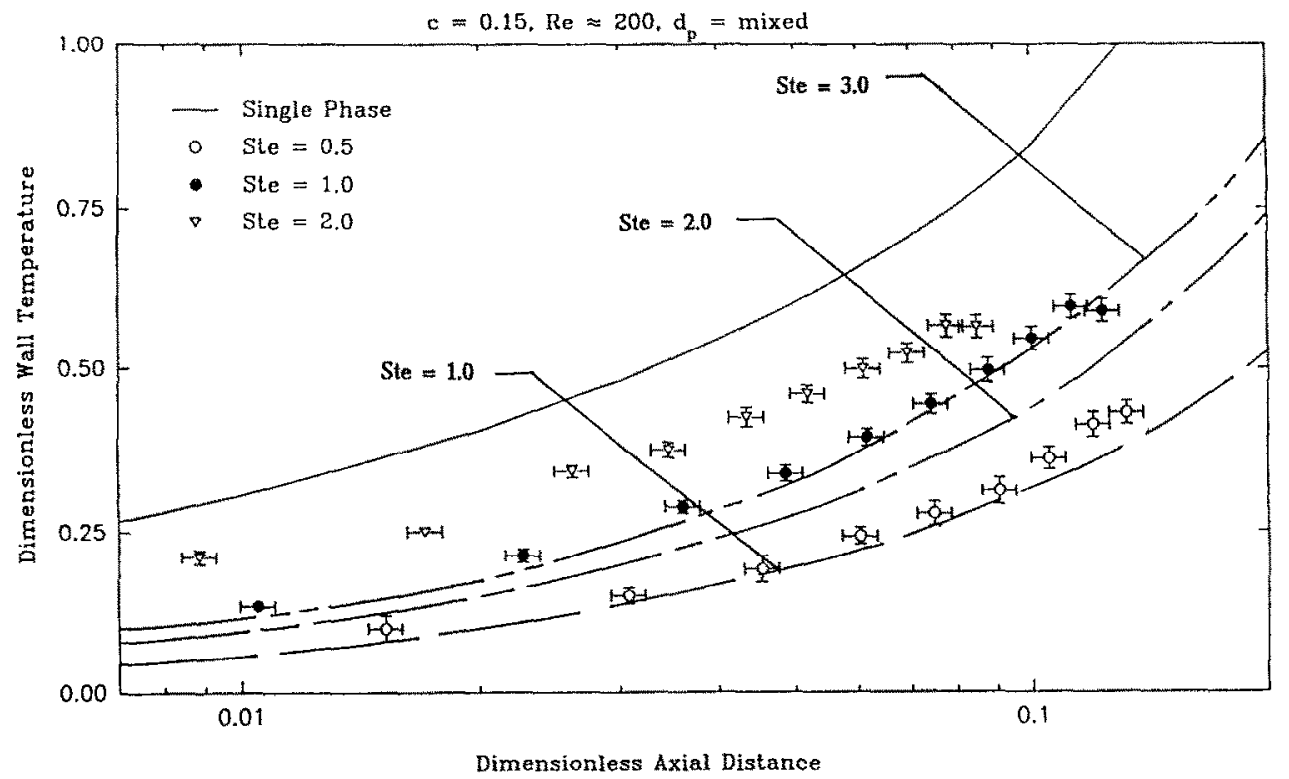

FiG. 5. Effect of bulk Stefan number on wall temperatures: comparison with numerical predictions from ref. [13] after accounting for sensible heat effects at inlet $(c=0.1)$.

temperatures approach the pure fluid case as the Stefan number (or heat flux) is increased. Thus, the greatest reduction in wall temperature rise is observed for Ste $=0.5$, which confirms Charunyakorn et al.'s [13] predictions regarding the effect of Stefan number. However, the reductions achieved in the experiments are not as large as those predicted by this study. This could have been due to a combination of a number of factors.

(a) Charunyakorn et al. [13] assumed that the suspension enters the test section at the melting tem- perature of the phase change material. In the experiments, the temperature of suspension entering the test section was slightly below the melting temperature, thus requiring some initial sensible heating before the actual phase change process could take place.

(b) Charunyakorn et al.'s [13] model assumes all the melting occurs exactly at the melting point of the phase change material. In practice, melting of the phase change material in the microcapsules takes place over a range of temperatures [16].

(c) Charunyakorn et al. [13] also assumed that

Ste $=1.0, \operatorname{Re} \approx 200$

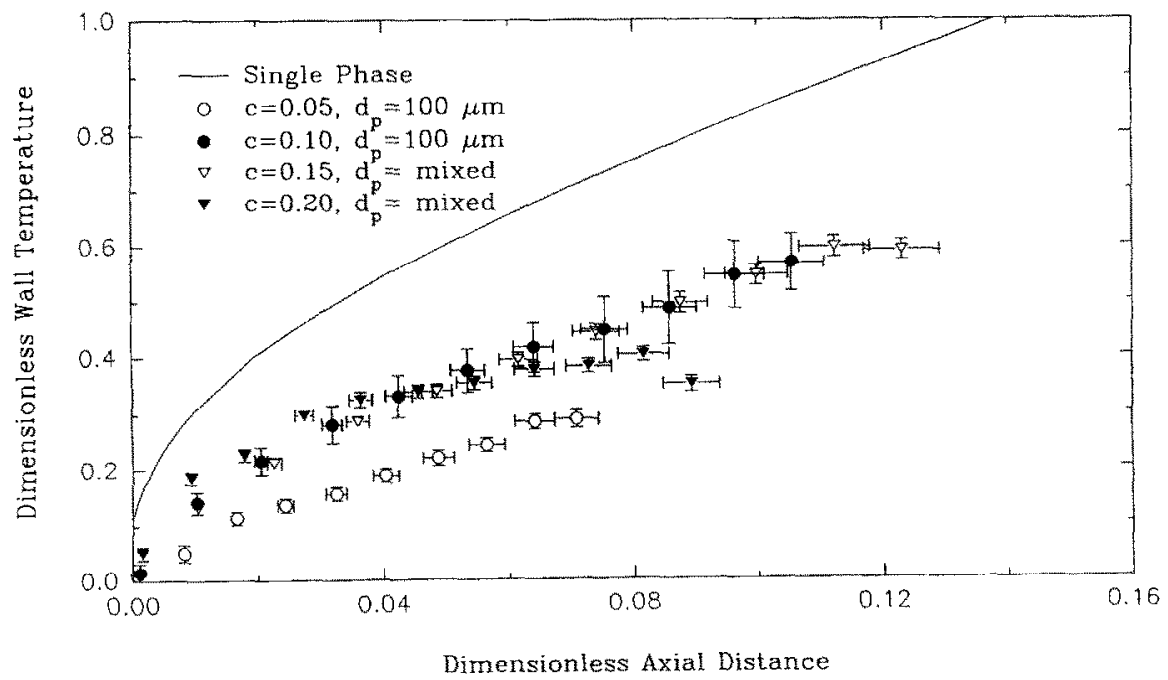

Fic. 6. Effect of volumetric concentration on wall temperatures (inlet condition location to account for initial subcooling). 
$c=0.05$, Ste $=1.0$, Re $\approx 200$

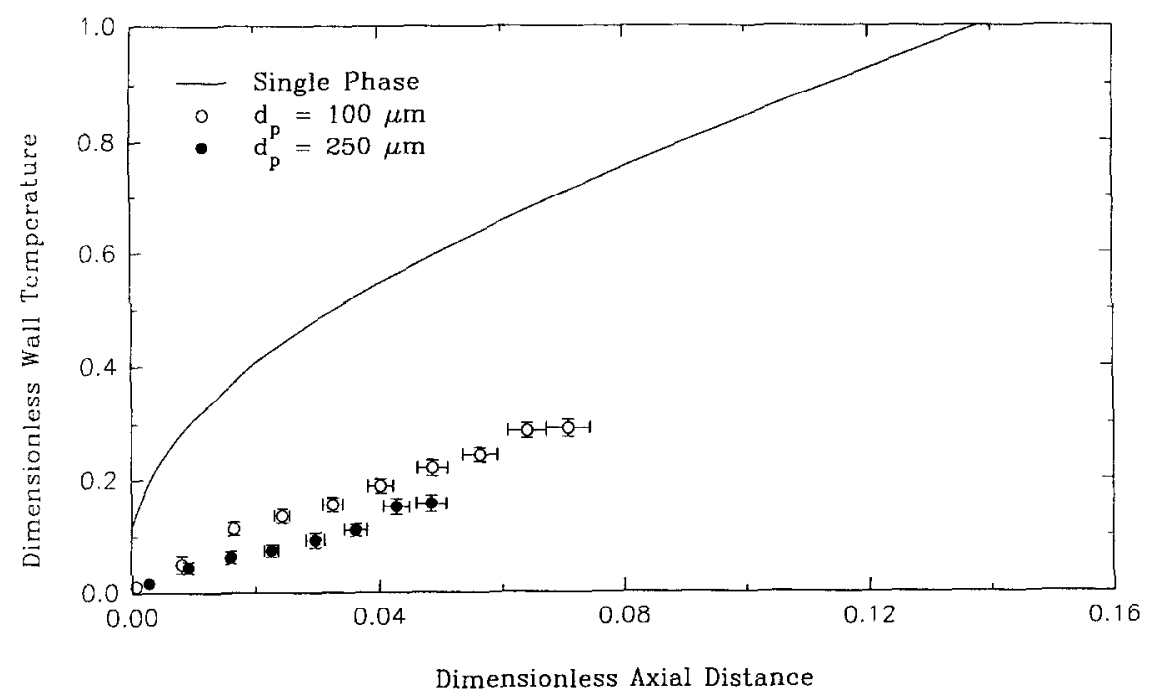

FIG. 7. Effect of microcapsule diameter on wall temperatures (initial location shifted to account for initial subcooling).

the microcapsules consist only of the phase change material. In the experiments this was not true since the phase change material was encapsulated in a wall which made up $30 \%$ of the weight of the microcapsule.

(d) Charunyakorn et al.'s [13] assumptions of a neutrally buoyant suspension and negligible radial migration of the microcapsules due to fluid-particle interactions (e.g. [24]) were not valid for this experimental study. In practice, radial migration combined with bunyancy induced separation affects the velocity profile and the homogeneity of the suspension, which in turn could affect the heat transfer.

The simplest correction is to account for the effec- tive concentration of the phase change material by comparing the results for $15 \%$ concentration experiments to Charunyakorn et al.'s [13] $10 \%$ concentration results so that the effective phase change material concentrations are similar (though not exactly identical). However, since the effects of concentration are small as discussed in the next section, this correction does not affect the overall results significantly.

Differences between the theoretical predictions and the experimental results due to factor (a) above, namely the effects of initial subcooling of the phase change material and consequent sensible heating of the suspension in the initial portion of the test section,

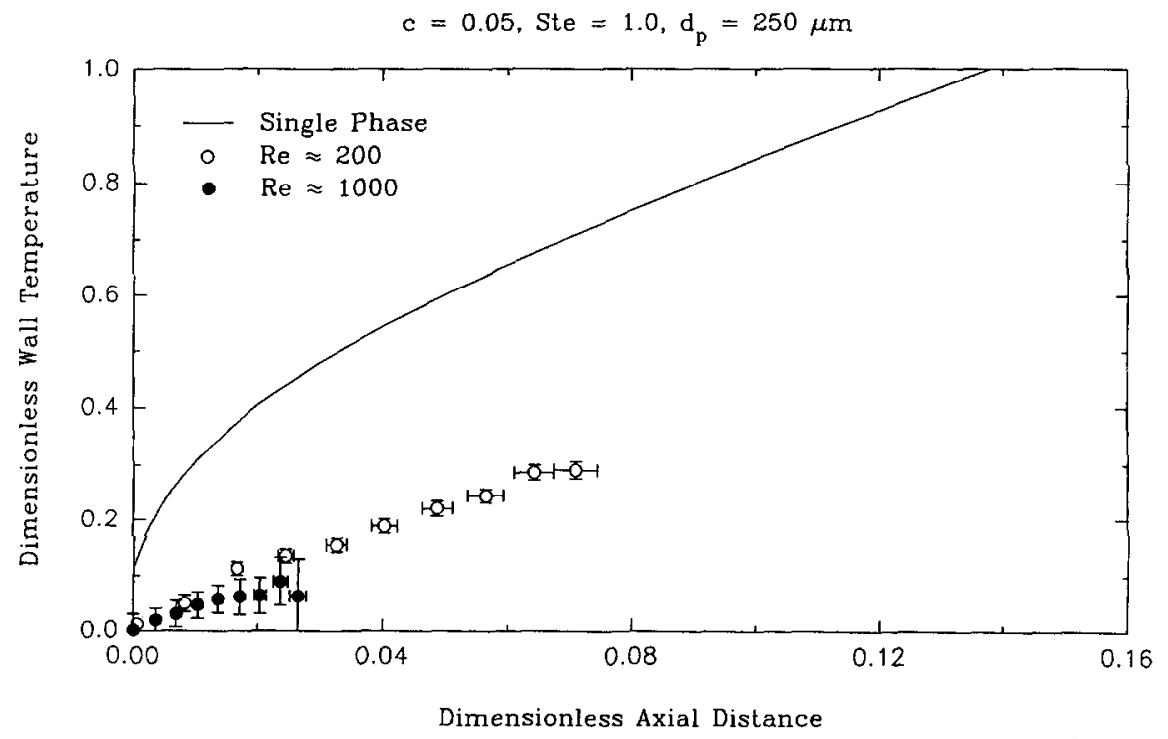

Fig. 8. Effect of flow rate on wall temperatures (inlet location shifted to account for initial subcooling). 


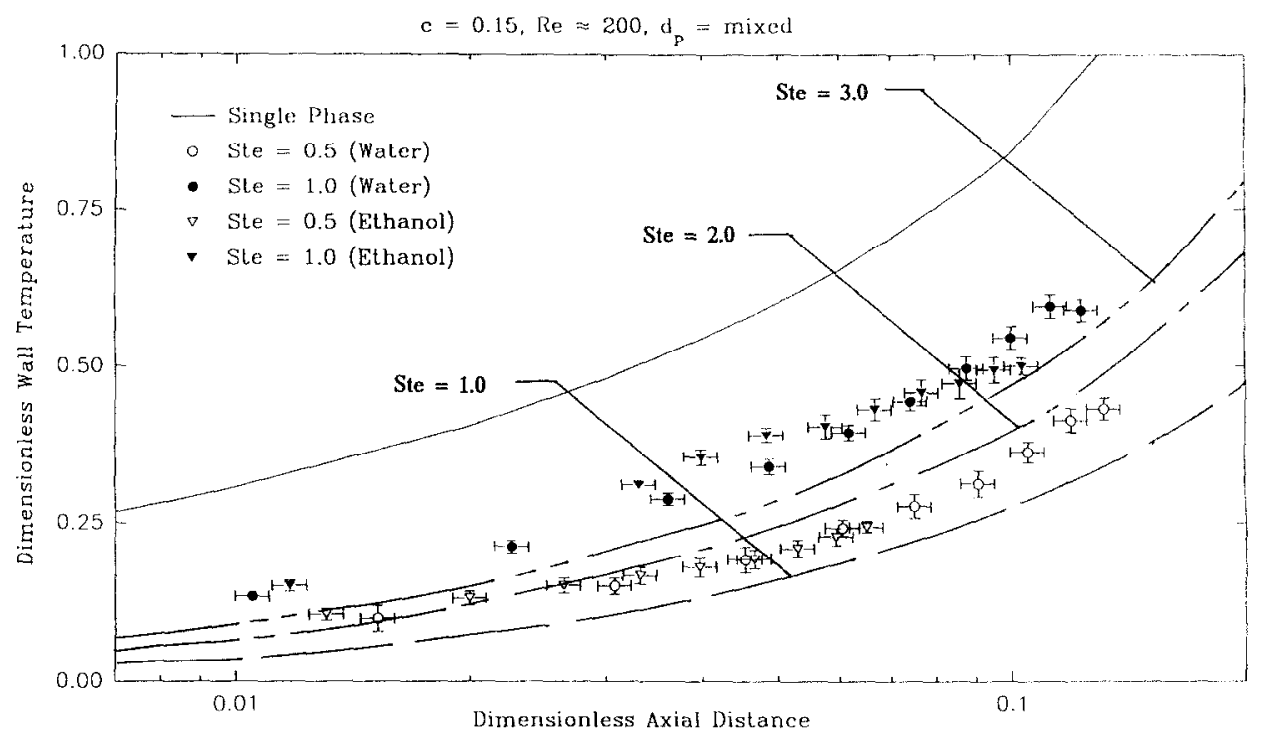

Fiv. 9. Comparison of wall temperatures for experiments with different suspending fluids (inlet condition shifted to account for initial subcooling).

can also be accounted for by a relatively simple correction. This was done by considering only the length of the test-section where the temperature was equal to or greater than the melting point of $n$-eicosane $\left(36.7^{\circ} \mathrm{C}\right)$. The location of the point where the suspension reached the melting temperature was obtained by a linear interpolation considering the two wall temperatures that were just below and above the melting temperature of the phase change material. Note that this correction procedure is approximate since it assumes that the entire flow reached the melting temperature at the same location. Furthermore, it does not include the cffect of varying melting temperature. Based on this approximation, the nondimensional temperature and length along the tube can be written as :

$$
\left(\theta_{\mathrm{wx}}\right)_{\mathrm{corr}}=\frac{T_{\mathrm{wx}}-36.7}{\frac{q_{\mathrm{w}} r_{\mathrm{d}}}{k_{\mathrm{b}}}}, \quad \bar{x}_{\mathrm{corr}}=\frac{x-x_{36.7}}{r_{\mathrm{d}} \operatorname{RePr}} .
$$

Using this correction, the difference between the experimental results and Charunyakorn et al.'s predictions is seen to be about $45 \%$ (Fig. 5). The effect of increasing Stefan number is also seen to be clearly much more pronounced, and the temperature rise is much slower when compared to the uncorrected temperature distribution. The difference between the two analyses is also larger for the lower Stefan number cases. Given the low heat inputs, this is expected because the overall temperature rise in these cases were small, and sensible heat effects become comparable to latent heat effects.

The effects of (d), which lead to a non-parabolic velocity profile and possibly a non-Newtonian type flow, cannot be quantified without a detailed analysis. This point coupled with the issues related to a varying melting temperature of the phase change material makes it inevitable that there will be some differences between Charunyakorn et al.'s [13] results and the present study. Further discussions on this issue follow in a later section.

\section{Effect of concentration}

The effect of concentration for a given Stefan number was found to be negligible except for very low concentrations (Fig. 6). The reason for the better performance for a concentration of 0.05 is unclear at the present time. It is important to note however, that concentration does play an indirect role in heat transfer through the bulk Stefan number. From the definition of the Stefan number, it is clear that for a given duct geometry, fluid and phase change material, its value depends only on the ratio of the wall heat flux to the volumetric concentration. Thus, for best cooling results, a low Stefan number should be maintained as far as possible, and concentration should be increased only if the heat flux increases.

\section{Effect of microcapsule diameter}

Particle diameter was studied as a parameter for only the 5 and $10 \%$ concentration cases. Figure 7 shows the effect of particle diameter for given concentrations and Stefan numbers. Prior to the experiments, based on Charunyakorn et al.'s work [13], it was believed that effect of particle diameter would be small. However, the experimental data show that the wall temperatures with the $250 \mu \mathrm{m}$ microcapsules are as much as $15 \%$ lower when compared to those with the $100 \mu \mathrm{m}$ microcapsules. A possible reason for this behavior is the separaton of the microcapsules in the suspension by the time it exits the test section. The microcapsules migrate towards the upper half of the 
tube due to buoyancy effects and could create an insulating layer once they are melted. Since the larger size of the $250 \mu \mathrm{m}$ microcapsules allows a lower packing density at the wall, this insulating effect would be smaller for larger particles as the suspending fluid could transport heat away from the wall more efficiently. Another possible reason is related to the radial migration of the microcapsules (e.g. [26]) which would be more prevalent for the $250 \mu \mathrm{m}$ microcapsules. For the present geometry this would have an opposing effect to the buoyancy forces, and once again reduce the insulating effect for the $250 \mu \mathrm{m}$ microcapsules.

\section{Effects of suspension homogeneity}

Two types of experiments were done to evaluate the effects of suspension homogeneity, first by considering cases with higher flow rates so that the degree of separation of the microcapsules could be reduced, and second by making a neutrally buoyant suspension using a $80 \%$ aqueous ethanol solution as the suspending fluid. The effect of flow rate of the suspension could be studied only for the $5 \%$ concentration experiments due to limited volumes of the suspension available. Figure 8 shows the effect of flow rate on the wall temperatures for given concentrations and Stefan numbers. It is observed that the effect of flow rate is negligible.

Experiments with a neutrally buoyant suspension were conducted for Stefan numbers 0.5 and 1.0 and a concentration of $15 \%$. Further experiments were not done because of the obvious damage to the microcapsules due to its interaction with the suspending fluid. Results of the neutrally buoyant solution compared to the original $n$-eicosane in water suspension results (Fig. 9) show that results for both neutrally and non-neutrally buoyant solutions are comparable. This, combined with the results for experiments to study the effects of flow rate suggests that the degree of homogeneity of the suspension does not have a significant effect on the heat transfer characteristics of the suspension. Based on this observation, it would seem that the velocity profile of the suspension is probably not very crucial in determining the heat transfer characteristics of the suspension. Thus, the primary reason for the differences between Charunykorn et al.'s [13] predictions and the experimental results is probably related to the varying melting temperature of the phase change material and not the nature of the flow field. Any modifications to Charunykorn et al.'s [13] theoretical model should therefore be directed primarily to this issue.

\section{Design considerations}

Based on the results of the experimental study, it is clear that wall temperature reductions of as much as $50 \%$ may be possible by using a phase change material suspension as the heat transfer fluid. The experiments were conducted with heat fluxes such that the wall temperature rise was $5-20^{\circ} \mathrm{C}$, which is representative of wall temperature rises where phase change material suspensions can be expected to show the best performance $v i s-\dot{a}$-vis a pure fluid. Results show that the relative reductions are high for low temperature rises, whereas it is lower for cases with high temperature rises. It is important to note that the heat fluxes involved were necessarily small in this case since only laminar flows were being considered. For turbulent flows, higher heat fluxes with similar reductions in wall temperatures may be possible since the effective bulk Stefan number based on temperature rise (which is more appropriate since it characterizes the sensible heat to the latent heat ratio more accurately) will be similar in both cases. No systematic studies, either theoretical or experimental, have been done for such flows. Finally, extrapolation of the experimental data to evaluate pumping power requirements shows even greater benefits in using phase change material suspensions. For example, considering Fig. 5, it can be seen that for the same temperature rises, the nondimensional axial distance can be increased by up to 10 times. Thus, for a given heat flux, the flow rate can be reduced to one-tenth (or by $90 \%$ ) and the pumping power to one-hundredth (or by 99\%). Similar reductions in pumping power have also been reported previously by Colvin and Mulligan [7, 8].

\section{CONCLUSIONS}

An experimental study has been conducted in order to evaluate the laminar flow heat transfer characteristics of a microencapsulated phase change material suspension flowing in a circular duct with constant wall heat flux. The primary parameters in this study were the bulk Stefan number and the volumetric concentration. In addition a limited number of experiments were done to evaluate the effects of the ductto-particle diameter ratio and the homogeneity of the suspension.

The bulk Stefan number, which is the ratio of the sensible heat capacity of the suspension to its latent heat capacily, was found to be the most dominant parameter. The volumetric concentration does not have a significant effect directly on the heat transfer. However, it is included in the definition of the Stefan number, and thus has an indirect effect. The microcapsule to duct diameter ratio had a noticeable effect on the heat transfer, but only limited results are available. The effect of the degree of homogeneity of the suspension was also found to be negligible, but once again, this result requires further experimental confirmation. These results agree qualitatively with the theoretical predictions of Charunyakorn et al. [13], but quantitative agreement is not very good. The probable reason for this is the varying melting temperature of the phase change material [16], and this point should be accounted for in any modified theoretical model.

Results show that up to $50 \%$ reduction in the wall temperature rise can be obtained by using micro- 
encapsulated phase change suspension in place of a single phase fluid. For best results, the bulk Stefan number should be maintained low (Ste $\leqslant 1.0$ ). This can be achieved either by selecting a phase change material such that its ratio of latent to sensible heat capacity is high, by increasing the concentration of the microcapsules or by reducing the duct radius. Finally, an important factor affecting the heat transfer characteristics was the inlet temperature or the initial subcooling of the incoming liquid. For inlet temperatures lower than the melting temperature of the phase change material, temperature reduction due to energy storage was overshadowed by the sensible heating process till the melting temperature in the fluid was reached.

Acknowledgements--The authors would like to acknowledge the financial support given by the Florida High Technology and Industry Council for this and related research through grants SUSF FHTIC-578 and FHTIC-644 and the National Science Foundation through grant NSF-CTS 027695.

\section{REFERENCES}

I. K. E. Kasza and M. M. Chen, Development of enhanced heal transfer/transport/storage sturries for thermalsystem improvement, ANL-82-50, Argonne National Lab., Illinois (1982).

2. H. G. Leal, On the effective conductivity of dilute sus pensions of spherical drops in the limit of low Peclet number, Chem. Engng Commun. 1, 2131 (1973).

3. R. Hart and F. Thornton, Microencapsulation of phase change materials, Final Report Contract No. 82-80, Ohio Department of Energy, Ohio (1982).

4. E. M. Mehalick and A. T. Tweedie, Two component thermal energy storage material, Report NSF/RANN/ SE/AER-74-09186, National Science Foundation, Washington, D.C. (1975)

5. P. A. Bahrami, Fusible pellet transport and storage of heat, Paper 82-HT-32, ASME, NY (1982).

6. W. A. McMahon, Jr., W. W. Harlowe, Jr. and D. J. Mangold, Feasibilty study of utilizing phase change coolant for protective garment, Final Report Contract No. DAAK60-81-C-0098, U.S. Army Natick Research and Development Command, MA (1982).

7. D. P. Colvin and J. C. Mulligan, Microencapsulated phase-change for storage of heat, NASA Tech Brief MFSA-27198, George C. Marshall Space Flight Center. AL (1987).

8. D. P. Colvin and J. C. Mulligan, Spacecraft heat rejection methods: active and passive heat transfer for electronic systems--Phase I, AFWAL-TR-86-3074, Flight Dynamics Laboratory, Air Force Wright Aeronautical Laboratories, $\mathrm{OH}$ (1986).

9. D. P. Colvin, Y. B. Bryant, J. C. Mulligan and J. D. Duncan, Microencapsulated phase change heat transfer systems. WRDC-TR-89-3072, U.S. Air Force Wright R\&D Center, OH (1989).

10. K. Chen and M. M. Chen, An analytical and experimental investigation of the convective heat transfer of phase change suspension flows. In Proc. of the International Symposium on Multiphase Flow (II), pp. 496 501. Zhejiang University Press, Zhejiang (1987).

11. D. P. Colvin and J. C. Mulligan. Method for using a PCM suspension to enhance heat transfer in liquids, U.S. Patent 4911232 (1990).

12. P. Charunyakorn, S. Sengupta and S. K. Roy, Forced convection heat transfer in microencapsulated phase change material slurries : flow between parallel plates. In General Papers: Phase Change and Convective Heal Transfer, ASME IITD-Vol. 129, pp. 55 62. ASME. NY (1990).

13. P. Charunyakorn, S. Sengupta and S. K. Roy, Forced convection heat transfer in microencapsulated phase change material slurries: flow in circular ducts, Int. I. Heat Mass Transfer 34, 819-835 (1991).

14. L. Katz. Natural convection heat transfer with fluids using suspended particles which undergo phase change, Ph.D. Dissertation, Massachusetts Institute of Technology, Massachusetts (1967)

15. P. Datta, S. Sengupta and S. K. Roy, Natural convection heat transfer in an enclosure with suspensions of microencapsulated phase change materials. In General Papers in Heat Transfer, HTD-Vol. 204. pp. 133 144. ASME. NY (1992)

16. S. K. Roy and S. Sengupta, An evaluation of phase change microcapsules for use in enhanced heat transfer fluids, Int. Commun. Heat Mass Transfer 18, 495-50/ (1991).

17. M. Goel, Laminar forced convection heat transfer in microencapsulated phase change material suspensions. M.S.M.E. Thesis, University of Miami, FL (1992).

18. E. C. Guyer and D. L. Brownell, Handbook of Applied Thermal Design. McGraw-Hill, New York (1988).

19. V. Vand, Theory of viscosity of concentrated suspensions, Nature 155, 364-365 (1945).

20. Ir. R. Rutgers, Relative viscosity of suspensions of rigid spheres in Newtonian liquids, Rheologica Acta 2, 305 348 (1962).

21. J. C. Maxwell, A Treatise on Electricity and Magnetism. Vol. 1, pp. 440-441. Dover, New York (1954).

22. E. Yamada and K. Takahashi, Effective thermal conductivity of suspensions-1st Report, Heat Transfer Jap. Res. 4, 83-101 (1975).

23. E. O. Doebelin, Measurement Svstems: Application and Design, Chap. 3. McGraw-Hill, New York (1966).

24. R. G. Cox and S. G. Mason, Suspended particles in fluid now through tubes, Ann. Rev. Fluid Mech. 4, 291-316 (I970).

25. Carrier Airconditioning Co., Handbook of Air Conditioning Systems. McGraw-Hill, New York (1965)

26. D. V. Hale, M. J. Hoover and M. J. O'Neil, Phasen Change Materials Handbook, NASA CR-61363 (1973).

27. W. J. Roff, J. R. Scott and J. Pacitti, Handbook of Common Polymers. CRC Press, Cleveland (1971). 